01

\title{
Совместное влияние магнитного поля и спин-поляризованного тока на связанную динамику магнитных вихрей в спин-трансферном наноосцилляторе
}

\author{
(C) Е.Г. Екомасов ${ }^{1-3}$, С.В. Степанов ${ }^{3}$, В.Н. Назаров ${ }^{4}$, К.А. Звездин ${ }^{5}$, Н.Г. Пугач 6,7 , Г.И. Антонов ${ }^{3}$ \\ ${ }^{1}$ Тюменский государственный университет, Тюмень, Россия \\ ${ }^{2}$ Башкирский государственный педагогический университет им. М. Акмуллы, Уфра, Россия \\ 3 Башкирский государственный университет, Уфа, Россия \\ ${ }^{4}$ Институт фризики молекул и кристаллов Уфимского федерального исследовательского центра РАН, Уфра, Россия \\ ${ }^{5}$ Институт общей физики им. А.М. Прохорова РАН, Москва, Россия \\ ${ }^{6}$ Московский государственный университет им. М.В. Ломоносова, Москва, Россия \\ ${ }^{7}$ Национальный исследовательский университет „Высшая школа экономики“, Москва, Россия \\ E-mail: EkomasovEG@gmail.com
}

Поступило в Редакцию 30 апреля 2021 г.

В окончательной редакции 24 мая 2021 r.

Принято к публикации 24 мая 2021 г.

\begin{abstract}
Исследуется совместное влияние спин-поляризованного тока и внешнего магнитного поля на динамику намагниченности в вихревых спин-трансферных наноосцилляторах диаметром $400 \mathrm{~nm}$. Для численного расчета связанной динамики магнитных вихрей использован пакет программ для микромагнитного моделирования SpinPM. Найдена зависимость от величины магнитного поля частоты стационарных связанных колебаний вихрей, определяющая рабочий диапазон частот перестраиваемого вихревого спин-трансферного наноосциллятора.
\end{abstract}

Ключевые слова: магнитные вихри, спин-трансферный наноосциллятор, магнитные нанодиски.

DOI: 10.21883/PJTF.2021.17.51382.18853

В настоящее время одним из перспективных технических устройств для спинтроники является микроволновой спин-трансферный наноосциллятор (СТНО) [1,2]. В его основе обычно лежат пермаллоевые наностолбики, которые имеют два магнитных слоя, разделенных немагнитной прослойкой $[2,3]$. В магнитных слоях как основное состояние может существовать магнитный вихрь [4]. Достаточно хорошо изучен случай, когда магнитный вихрь существует лишь в одном из слоев (см., например, [4,5]). Показано, что для работы вихревого СТНО удобно использовать режим стационарно движущегося вихря. Частотой таких осцилляций можно управлять с помощью внешнего магнитного поля и спин-поляризованного тока. В вихревых СТНО удалось достичь высокой мощности микроволнового сигнала (порядка $1 \mu \mathrm{W}$ ) и сравнительно узкой ширины линии (менее $0.2 \mathrm{MHz}$ ).

Гораздо менее изучен случай, когда магнитный вихрь существует в каждом из слоев [6,7]. Теоретически показано, что с помощью спин-поляризованного тока можно возбудить гиротропные магнитостатически связанные колебания вихрей с постоянной частотой, которая может меняться при приложении магнитного поля $[8,9]$. Экспериментально построена зависимость частоты гиротропных связанных колебаний вихрей от величины постоянного магнитного поля, перпендикулярного плоскости дисков [10]. Показано, что при определенном сочетании толщины и диаметра диска, величины спин- поляризованного тока и магнитного поля магнитный вихрь может вылетать за пределы диска [8], что является проблемой для устойчивой работы вихревого СТНО. $\mathrm{B}$ настоящей работе исследуется совместное влияние внешнего магнитного поля, перпендикулярного плоскости диска, и тока на динамику связанных вихрей в СТНО большого диаметра, когда магнитный вихрь не может вылететь за край диска.

Рассмотрим наностолбик кругового сечения диаметром $400 \mathrm{~nm}$. Он содержит три слоя: толстый магнитный слой из пермаллоя (толщина $15 \mathrm{~nm}$ ), промежуточный немагнитный слой (толщина $10 \mathrm{~nm}$ ) и тонкий магнитный слой из пермаллоя (толщина $4 \mathrm{~nm}$ ). Магнитные параметры системы следующие: намагниченность насыщения $M_{s}=700 \mathrm{erg} /\left(\mathrm{G} \cdot \mathrm{cm}^{3}\right)$ для толстого и $600 \mathrm{erg} /\left(\mathrm{G} \cdot \mathrm{cm}^{3}\right)$ для тонкого слоя, обменная жесткость $A=1.2 \cdot 10^{-6} \mathrm{erg} / \mathrm{cm}$ для толстого и $1.12 \cdot 10^{-6} \mathrm{erg} / \mathrm{cm}$ для тонкого слоя, $\alpha=0.01, \gamma=2.0023(\mathrm{erg} \cdot \mathrm{s})^{-1}$ [7]. Нелинейную динамику намагниченности в магнитном слое будем описывать с помощью обобщенного уравнения Ландау-Лифшица (ОУЛЛ). Оно содержит дополнительный вращательный момент [3], ответственный за взаимодействие тока с намагниченностью, и имеет вид

$$
\dot{\mathbf{M}}=-\gamma\left[\mathbf{M} \times \mathbf{H}_{e f f}\right]+\frac{\alpha}{M_{s}}[\mathbf{M} \times \dot{\mathbf{M}}]+\mathbf{T}_{\text {s.t. }},
$$

где $\mathbf{M}$ - вектор намагниченности, $\gamma$ - гиромагнитное отношение, $\alpha$ - параметр затухания Гильберта. Эффективное поле $\mathbf{H}_{e f f}$ представляет собой сумму внешнего 
магнитного поля, полей магнитостатического и обменного взаимодействий. Дополнительный вращательный момент $\mathbf{T}_{\text {s.t. }}$ в уравнении (1) линейно зависит от величины плотности тока и его поляризации [3] и состоит из двух компонент: параллельной и перпендикулярной плоскости $\left(\mathbf{M}, \mathbf{m}_{r e f}\right)$, где $\mathbf{m}_{r e f}-$ единичный вектор вдоль намагниченности опорного слоя. Обобщенное уравнение Ландау-Лифшица в общем случае представляет собой интегро-дифференциальное уравнение.

Для численного расчета связанной динамики магнитных вихрей использован пакет программ для микромагнитного моделирования SpinPM [7,11]. Будем исследовать динамику двух связанных магнитных вихрей с одинаковой полярностью (П-вихри) и киральностью, существующих в начальный момент времени в каждом магнитном слое. Магнитные вихри с разной полярностью будем называть АП-вихрями. Пусть ток течет снизу вверх перпендикулярно плоскости слоев (от толстого слоя к тонкому). Поляризацию тока возьмем равной $P=0.1$, начальные намагниченности в центре вихрей направлены вверх.

С помощью микромагнитного моделирования в зависимости от величины приложенного тока выявлены три сценария динамики вихря. При малых величинах плотности токов наблюдаются затухающие колебания вихря. При средних значениях плотности токов имеет место стационарный режим колебаний вихря с постоянной частотой, зависящей от параметров системы и величины тока. При больших значениях плотности токов наблюдается динамическое переключение полярности вихря в толстом слое, сопровождающееся сложной трансформацией структуры исходного вихря. Далее образуются АП-вихри и наблюдается стационарный режим их связанных колебаний. В [11] для случая нулевого магнитного поля найден диапазон частот стационарного режима колебаний П-вихрей и АП-вихрей. Величина первой критической плотности тока (начала стационарного режима колебаний П-вихрей) равна $j=4.9 \cdot 10^{7} \mathrm{~A} / \mathrm{cm}^{2}$, величина второй критической плотности тока (начала

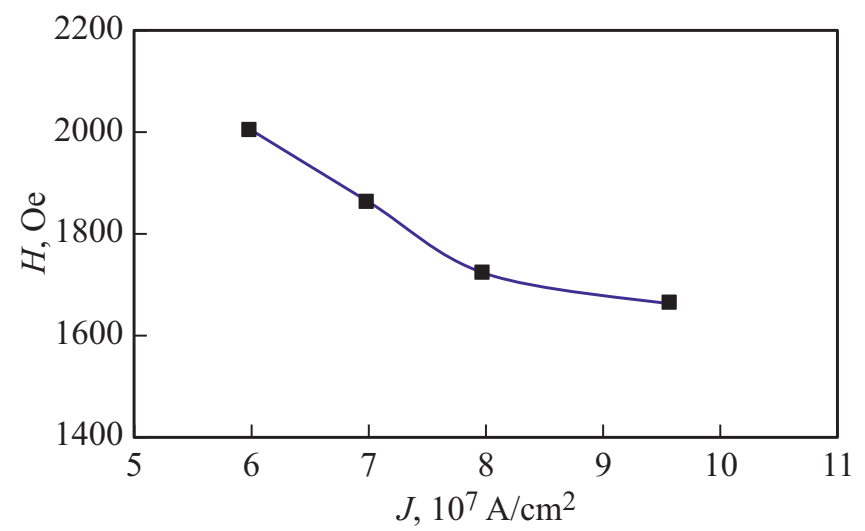

Рис. 1. Зависимость критической величины магнитного поля от плотности тока для случая стационарных связанных колебаний магнитных П-вихрей.

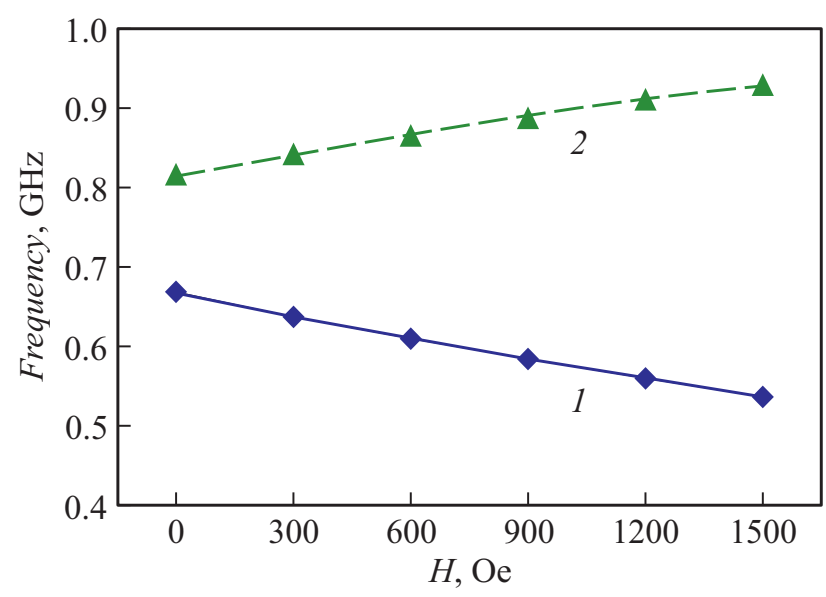

Рис. 2. Зависимость частоты связанных колебаний магнитных вихрей от величины внешнего перпендикулярного плоскости диска магнитного поля. $j=6 \cdot 10^{7}(1)$ и $10 \cdot 10^{7} \mathrm{~A} / \mathrm{cm}^{2}(2)$.

стационарного режима колебаний АП-вихрей) равна $j=9.6 \cdot 10^{7} \mathrm{~A} / \mathrm{cm}^{2}$. Далее исследуем совместное влияние спин-поляризованного тока и внешнего магнитного поля, перпендикулярного плоскости диска, на стационарную динамику вихрей. С помощью микромагнитного моделирования определены величины критического магнитного поля, ниже которого при данном значении плотности тока еще возможен стационарный режим колебаний П-вихрей и АП-вихрей (рис. 1). Для полученного диапазона возможных значений величин полей и токов найдена зависимость частоты стационарного связанного колебания вихрей от внешнего магнитного поля при постоянном значении тока (рис. 2). При расчетах использовалось два значения плотности тока. При первом значении плотности тока, равном $6 \cdot 10^{7} \mathrm{~A} / \mathrm{cm}^{2}$, имеем изначально П-вихри, при втором значении плотности тока, равном $10 \cdot 10^{7} \mathrm{~A} / \mathrm{cm}^{2}$, имеем изначально АП-вихри. Видно, что полученные численно зависимости в обоих случаях, как и для случая одиночного вихря, полученного ранее [5], можно аппроксимировать линейной функцией

$$
\omega=\omega_{0}(1+k H),
$$

где $k$ - коэффициент пропорциональности, $\omega_{0}$ - частота колебаний в случае нулевого магнитного поля. Отметим, что с помощью магнитного поля в рассмотренных случаях удается получить изменение частоты колебаний вихревого СТНО приблизительно на 20\%. Зависимость частоты от магнитного поля, слабо отличающаяся от линейной, была получена ранее аналитически с помощью использования уравнений Тиля для случая слабой магнитостатической связи вихрей [9]. Сравнение полученных результатов со случаем СТНО меньшего диаметра $[8,9]$ указывает на то, что с увеличением диаметра диска происходит уменьшение величины коэффициента $k$ в формуле (2). Подобная линейная зависимость частоты стационарного движения связанных вихрей от внешнего 
перпендикулярного магнитного поля была получена экспериментально для случая АП-вихрей в пермаллоевом нанодиске диаметром $250 \mathrm{~nm}$ [10]. В этой работе также показано, что экспериментально пока невозможно получить информацию о стационарной динамике П-вихрей. Поэтому теоретические результаты, полученные в настоящей работе, важны при проектировании вихревых СТНО для практических приложений.

В заключение отметим, что нами описана возможность управления рабочей частотой перестраиваемого вихревого СТНО в широком диапазоне с помощью внешнего магнитного поля и спин-поляризованного тока. Показано, что для вихревого СТНО диаметром $400 \mathrm{~nm}$ использование стационарного режима колебаний вихрей, с одной стороны, приводит к уменьшению частоты колебаний по сравнению со случаями среднего и малого диаметров, но, с другой стороны, случай диаметра $400 \mathrm{~nm}$ является более предпочтительным, так как не приводит к нежелательному вылету вихря за край диска.

\section{Финансирование работы}

Микромагнитное моделирование влияния спин-поляризованного тока на связанную динамику магнитных вихрей выполнено при финансовой поддержке Российского фонда фундаментальных исследований (проект № 19-02-00316), анализ совместного влияния внешнего магнитного поля и тока на частоту колебаний СТНО осуществлялся при поддержке ВШЭ и БГПУ в рамках проекта „Зеркальные лаборатории“.

\section{Конфликт интересов}

Авторы заявляют, что у них нет конфликта интересов.

\section{Список литературы}

[1] P.N. Skirdkov, A.F. Popkov, K.A. Zvezdin, Appl. Phys. Lett., 113, 242403 (2018). DOI: 10.1063/1.5064440

[2] A. Litvinenko, V. Iurchuk, P. Sethi, S. Louis, V. Tyberkevych, J. Li, A. Jenkins, R. Ferreira, B. Dieny, A. Slavin, U. Ebels, Nano Lett., 20, 6104 (2020).

DOI: 10.1021/acs.nanolett.0c02195

[3] А.К. Звездин, А.В. Хвальковский, К.А. Звездин, УФН, 178 (4), 436 (2008). DOI: 10.3367/UFNr.0178.200804i.0436 [Пер. версия: 10.1070/PU2008v051n04ABEH006508].

[4] A. Khvalkovskiy, J. Grollier, A. Dussaux, K.A. Zvezdin, V. Cros, Phys. Rev. B, 80, 140401 (2009). DOI: 10.1103/PhysRevB.80.140401

[5] П.Д. Ким, В.А. Орлов, В.С. Прокопенко, С.С. Замай, В.Я. Принц, Р.Ю. Руденко, Т.В. Руденко, ФТТ, 57 (1), 29 (2015). [Пер. версия: 10.1134/S1063783415010151].

[6] S.S. Cherepov, B.C. Koop, V. Korenivski, D.C. Worledge, A.Yu. Galkin, R.S. Khymyn, B.A. Ivanov, Phys. Rev. Lett., 109, 097204 (2012). DOI: 10.1103/PhysRevLett.109.097204

[7] N. Locatelli, A.E. Ekomasov, A.V. Khvalkovskiy, Sh.A. Azamatov, K.A. Zvezdin, J. Grollier, E.G. Ekomasov, V. Cros, Appl. Phys. Lett., 102, 062401 (2013).

DOI: $10.1063 / 1.4790841$
[8] А.Е. Екомасов, С.В. Степанов, К.А. Звездин, Е.Г. Екомасов, ФМM, 118 (4), 345 (2017). DOI: $10.7868 / \mathrm{S} 0015323017020024$ [Пер. версия: 10.1134/S0031918X17020028].

[9] С.В. Степанов, А.Е. Екомасов, К.А. Звездин, Е.Г. Екомасов, ФTT, 60 (6), 1045 (2018). DOI: $10.21883 /$ FTT.2018.06.45974.22M [Пер. версия: 10.1134/S1063783418060318].

[10] A. Hamadeh, N. Locatelli, V. Naletov, R. Lebrun, G. Loubens, J. Grollier, O. Klein, V. Cros, Phys. Rev. Lett., 112, 257201 (2014). DOI: 10.1103/PhysRevLett.112.257201

[11] Е.Г. Екомасов, С.В. Степанов, К.А. Звездин, Н.Г. Пугач, Г.И. Антонов, ФММ, 122 (3), 212 (2021). DOI: $10.31857 / \mathrm{S} 0015323021030050$ 